\title{
Hypercoagulopathy in COVID-19, Deep Venous Thrombosis After Extra-Corporeal Membrane Oxygenation Therapy: A Case Report
}

\author{
Akash Garg $^{\mathrm{a}}$, Himan Goli ${ }^{\mathrm{a}}$, Fariba Yazdanpanah ${ }^{\mathrm{a}, \mathrm{b}}$
}

\begin{abstract}
Severe acute respiratory syndrome coronavirus 2 (SARS-CoV-2) is a novel coronavirus that has caused a pandemic across the world in the years 2019 - 2020 with over 100 countries reporting high infection rates. The virus is unique in the wide spectrum of disease manifestations it can cause; one of the worst of which is the hypercoagulable state induced by severe infection. This case report focuses on a 33-year-old Hispanic male who developed severe acute respiratory syndrome requiring management with extracorporeal membrane oxygenation (ECMO) and developed deep venous thromboses during severe coronavirus disease 2019 (COVID-19) pneumonia. Since there are no current guideline(s) for routine screening for venous thromboembolism (VTE) in ECMO patients, we aim to highlight a proposed benefit of routine screening for VTE in patients with severe COVID-19 treated with ECMO pre-cannulation and post-decannulation, which minimizes the risk of cannulation-associated complications, as well as the risk of post-decannulation VTE respectively. While VTE is a known complication of ECMO therapy, the rates of increased incidence of VTE in patients with severe COVID-19 make the detection of such complications even more important to reduce overall morbidity and mortality.
\end{abstract}

Keywords: COVID-19; Severe acute respiratory syndrome coronavirus 2; Extracorporeal membrane oxygenation; Venous thromboembolism; Deep venous thrombosis; Hypercoagulopathy

\section{Introduction}

Severe acute respiratory syndrome coronavirus 2 (SARS-

Manuscript submitted February 2, 2021, accepted February 18, 2021

Published online March 24, 2021

aUniversity of Maryland Capital Region Health, 3001 Hospital Drive, Cheverly, MD 20785, USA

${ }^{b}$ Corresponding Author: Fariba Yazdanpanah, University of Maryland Capital Region Health, 3001 Hospital Drive, Cheverly, MD 20785, USA.

Email: Fariba.yazdanpanah@umm.edu

doi: https://doi.org/10.14740/jmc3674
CoV-2) is a novel coronavirus that has caused a pandemic across the world in the years 2019 - 2020 with over 100 countries reporting high infection rates $(>20,000$ cases) [1]. In addition to the significant infectivity of the virus, the disease caused by the virus, coronavirus disease 2019 (COVID-19), has demonstrated significant mortality. One of the most interesting observations about COVID-19 is the wide range of clinical manifestations it has from mild upper respiratory illness to respiratory failure and acute respiratory distress syndrome (ARDS). This report highlights one of these manifestations, namely the hypercoagulopathy suspected to be caused by SARS-CoV-2 infection as evidenced by multiple systematic reviews and case series [2]. As the number of cases rose exponentially early on in the pandemic, investigations were underway to understand the pathophysiological mechanisms for severe disease and ultimately death. Multiple autopsy reports demonstrated evidence for microvascular thrombosis in pulmonary vessels suspected to result from pneumonitis and alveolar injury induced by the infection $[3,4]$. In our case, we will demonstrate that SARS-CoV-2 infection is also associated with macrovascular thrombosis, and we will discuss the implications of this complication in the management of severe COVID-19 cases who require extracorporeal membrane oxygenation $(\mathrm{ECMO})$ therapy.

\section{Case Report}

\section{Investigations and diagnosis}

A 33-year-old Hispanic male with no past medical history presented initially with subjective fever (temperature $37.4^{\circ} \mathrm{C}$ ) and dry cough. He was diagnosed with COVID-19 pneumonia via positive "Xpert SARS-COVID 2 PCR" nasopharyngeal swab, and computed tomography (CT) angiography chest showing multifocal, patchy, and bilateral ground-glass opacities in a peripheral distribution without any evidence for pulmonary embolism (PE) (Fig. 1). Initially, our patient was sent home after an evaluation revealed no fever, mild tachycardia with a heart rate of 117 beats/min, mild tachypnea with a respiratory rate of 22 breaths/min, and no increased oxygen requirement. He was given a prescription for azithromycin $250 \mathrm{mg}$ daily and advice for home quarantining and supportive care. 


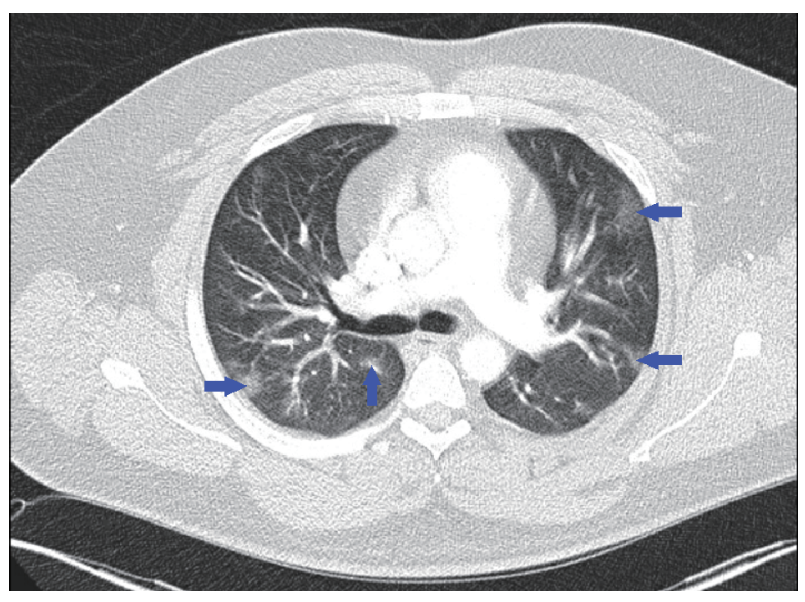

Figure 1. Chest computed tomography scan with intravenous contrast showing multifocal, patchy, and bilateral ground-glass opacities.

However, he returned 5 days later with worsening subjective fever, dry cough, and the addition of nausea, and shortness of breath. Evaluation at that time, revealed temperature 37.2 ${ }^{\circ} \mathrm{C}$, mild tachycardia with heart rate 118 beats/min, worsened tachypnea with respiratory rate $24-41$ breaths/min, oxygen saturation down to $86 \%$ on room air, up to $92 \%$ on $4 \mathrm{~L} / \mathrm{min}$ of oxygen via nasal cannula, but our patient continued to have respiratory distress and shortness of breath, which were relieved only with supplemental oxygen from high-flow nasal cannula set at fraction of inspired oxygen $100 \%$ with $25 \mathrm{~L} / \mathrm{min}$ of oxygen flow. Repeat CT angiography chest was completed, which again showed no evidence of PE, but interval increase of the bilateral pulmonary opacities noted on the previous CT 5 days before (Fig. 2). The patient was initially admitted to the medical/surgical (Med/Surg) telemetry ward.

Inflammatory markers, initially, showed significant elevations except for $\mathrm{D}$-dimer which was $0.460 \mu \mathrm{g}$ fibrinogen equivalent unit (FEU)/mL (normal range: 0 - $0.490 \mu \mathrm{g} \mathrm{FEU} / \mathrm{mL}$ ) (Creactive protein $(\mathrm{CRP})>300 \mathrm{mg} / \mathrm{L}$ (normal range: $0.5-5 \mathrm{mg} / \mathrm{L}$ ), ferritin $>2,000 \mathrm{ng} / \mathrm{mL}$ (normal range: $30-400 \mathrm{ng} / \mathrm{mL}$ ), lactate dehydrogenase (LDH) $492 \mathrm{U} / \mathrm{L}$ (normal range: 135 - $225 \mathrm{U} / \mathrm{L}$ ), fibrinogen 1,100 mg/dL (normal range: $240-510 \mathrm{mg} / \mathrm{dL}$ ), procalcitonin $0.9 \mathrm{ng} / \mathrm{mL}$ (normal range: 0 - $0.1 \mathrm{ng} / \mathrm{mL}$ )].

\section{Treatment}

The patient was being treated for possible superimposed bacterial pneumonia with empiric antibiotics as well as supportive antioxidant vitamins/minerals, and was initially receiving deep venous thrombosis (DVT) prophylaxis with enoxaparin $40 \mathrm{mg}$ subcutaneously daily. His supplemental oxygen requirements remained high, increasing from a flow rate of $25 \mathrm{~L} / \mathrm{min}$ to 35 $\mathrm{L} / \mathrm{min}$, and remaining on $100 \%$ fraction of inspired oxygen. Despite this respiratory support, he continued to desaturate as low as $88 \%$, eventually requiring intubation and mechanical ventilation before being transferred to a tertiary care facility to undergo central veno-venous (CVV)-ECMO.

After transfer, the patient was given convalescent plasma

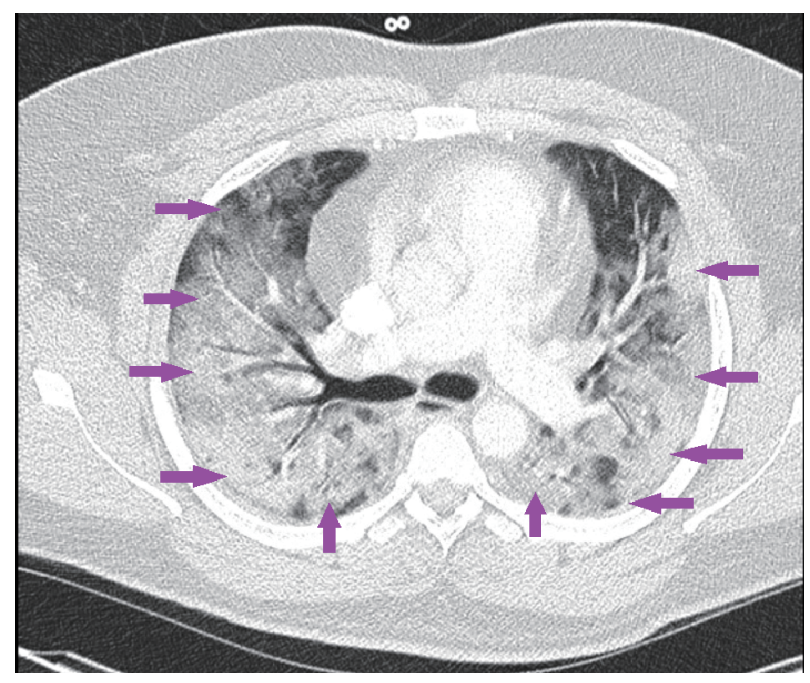

Figure 2. Repeat chest computed tomography scan with intravenous contrast showing interval increase in pulmonary opacities bilaterally without any evidence of pulmonary embolism.

and increasing doses of medical DVT prophylaxis with enoxaparin $40 \mathrm{mg}$ subcutaneously every $12 \mathrm{~h}$, as his D-dimer trended upward to $4.09 \mu \mathrm{g} \mathrm{FEU} / \mathrm{mL}$ from $0.460 \mu \mathrm{g} \mathrm{FEU} / \mathrm{mL}$ (normal range: $0-0.490 \mu \mathrm{g} F E U / \mathrm{mL}$ ). He was also cannulated for CVV-ECMO in his right internal jugular vein (RIJV) and right common femoral vein (RCFV). A left internal jugular central venous catheter was placed for continued care. As part of CVVECMO therapy, the patient received a continuous infusion of unfractionated heparin with a goal activated partial thromboplastin time (aPTT) of 60 - 80 throughout the whole time spent on ECMO and for one additional day. While on ECMO, the patient spent the majority of time within the target range.

During his hospital course, he underwent tracheostomy tube placement for failing to wean off of mechanical ventilation. Despite continuous therapeutic anticoagulation with unfractionated heparin, the patient's D-dimer continued to trend upward to a peak level of $17.79 \mu \mathrm{g} \mathrm{FEU} / \mathrm{mL}$ (normal range: $0-0.490 \mu \mathrm{g} F E U / \mathrm{mL})$. The day after decannulation, he underwent controlled ultrasound (US) venous duplex scans of the RIJV and RCFV, which revealed non-occlusive thrombi in both veins. Therapeutic dosing of anticoagulant therapy continued despite being off of CVV-ECMO given the discovery of these DVTs.

Repeat nasopharyngeal swab and tracheal aspirate tests for SARS-CoV-2 after 40 days were negative and the patient was subsequently transferred back to the community hospital for further care.

Eventually, after hospitalization for 58 days, the patient was discharged home on warfarin $7.5 \mathrm{mg}$ daily with prothrombin time/international normalized ratio (PT/INR) monitoring as an outpatient.

\section{Discussion}

COVID-19 disease is a novel presentation of Coronaviridae, 
which has caused a worldwide pandemic. In addition to the multi-system organ involvement, the virus can be associated with coagulopathy as well [2], which has been described in systematic reviews and a few case series with an increased incidence of VTE associated with COVID-19 disease up to $6.2 \%$ [5]. The underlying cause of the hypercoagulable state induced by SARS-CoV-2 infection is suspected to be the severe systemic inflammatory response [6]. The primary predictive marker associated with increased risk of VTE in COVID-19 patients is the D-dimer with a direct relationship between D-dimer level and increased incidence of VTE [7]. It should be noted that catheter-associated DVTs (CaDVT) are a known complication of CVV-ECMO, the incidence of which is reported to be in the range of $10-85 \%$ in patients without SARS-CoV-2 infection [8]. Therefore, it is important to report on instances of CaDVTs in the setting of COVID-19 due to the severe extent of the disease and because of the increasing use of ECMO in the treatment of severe COVID-19 patients.

Our case was appropriately started on CVV-ECMO for persisting hypoxic respiratory failure due to severe COVID-19 pneumonia. Immediately before the initiation of CVV-ECMO, the patient's D-dimer was $2.67 \mu \mathrm{g} F E U / m L$ (normal range: 0 $0.490 \mu \mathrm{g} \mathrm{FEU} / \mathrm{mL}$ ) and there was no evidence of VTE at that time. Despite appropriate practices to prevent thrombosis from SARS-CoV-2 and simultaneous ECMO therapy, the patient's D-dimer continued to trend upward, and the patient developed DVTs at the cannula sites in his RIJV and RCFV. Artifoni et al showed that higher levels of D-dimer correlate positively with a higher incidence of VTE in COVID-19 patients [7]. Subsequently, in our case, post-decannulation US venous duplex revealed DVTs in the RIJV and RCFV.

Based upon ongoing available research, a wide range of incidences (23-85.4\%) have been reported for CaDVT in the setting of ECMO therapy since 2017 [8-10]. Because of this inconsistency in incidence reports, there is currently no universal guideline for post-decannulation US scans to check for VTE at cannulation sites. Current recommendations from the Extracorporeal Life Support Organization (ELSO) are to screen patients based on clinical suspicion in each individual case [11]. However, given that COVID-19 disease is suspected to be associated with hypercoagulopathy and that there are already varying reports of CaDVT associated with ECMO therapy, some with rates alarmingly high more than $60 \%$ [10], protocols for ECMO therapy would benefit from the addition of routine post-decannulation screening recommendations. At least, during the current pandemic, patients with COVID-19 who require ECMO therapy would benefit from routine screening within $24-48 \mathrm{~h}$ post-decannulation to ensure that potentially life-threatening diagnoses are not missed.

Data indicating that the rates of using ECMO in the treatment of COVID-19 patients are scarce, although the ELSO registry shows that there are 3,360 confirmed COVID-19 cases currently receiving ECMO therapy [12]. Without having reliable data about the current number of active cases, which is difficult to ascertain because, while death rates are reported promptly, recovery rates are not, it is too complicated to establish an estimation of the rate of ECMO therapy usage in COVID-19 cases.

In conjunction with this difficulty, there are even fewer reports of VTE post-ECMO-decannulation. To our knowledge, there is a single article by Belys et al in the British Journal of Anaesthesiology, which describes 12 patients who were treated with CVV-ECMO for severe COVID-19 ARDS between March 1, 2020 and April 4, 2020 [13]. Of these, six patients were noted to have VTE, four of which were discovered during cannulation for CVV-ECMO, one of which developed massive $\mathrm{PE}$ as a result of cannulation and died, one of the six developed CaDVT, and the remaining two patients of six developed intra-circuit thrombosis [13]. The conclusion of this paper was that screening for VTE prior to the initiation of CVV-ECMO in COVID-19 ARDS patients should be done. However, since VTE is a known complication of CVV-ECMO by itself without the presence of SARS-CoV-2 infection, and since currently during the SARS-CoV-2 pandemic, the infection is strongly suspected to induce hypercoagulopathy, our case shows that screening before starting ECMO therapy would not be enough. Therefore, we argue that protocols should be written recommending screening for VTE with Doppler US both pre-cannulation and post-decannulation related to CVV-ECMO therapy in COVID-19 patients.

To our knowledge, hypercoagulopathy with COVID-19 disease is an uncommon occurrence based on the evidence currently available. However, the severity of the disease makes further research into this subject crucial especially since data regarding VTE in COVID-19 patients are increasing. In addition, as a large number of severe COVID-19 ARDS cases require ECMO therapy during the course of treatment, a protocol to screen patients for VTE post-ECMO-decannulation would be especially useful in this patient population given the high degree of suspicion that SARS-CoV-2 infection induces a hypercoagulable state.

\section{Learning points}

COVID-19 has many disease manifestations with growing evidence suggesting the disease induces a hypercoagulable state. Many COVID-19 cases are severe enough to warrant ECMO therapy during the course of treatment. ECMO therapy carries a risk of VTE by itself but this risk is likely worsened by COVID-19 with dire consequences. To mitigate these consequences, we recommend updating current protocols for managing COVID-19 patients who require ECMO therapy with pre-cannulation and post-decannulation US venous duplex to screen patients for possible VTE.

\section{Acknowledgments}

None to declare.

\section{Financial Disclosure}

None to declare. 


\section{Conflict of Interest}

None to declare.

\section{Informed Consent}

Not applicable.

\section{Author Contributions}

Dr. Garg and Dr. Yazdanpanah devised the project, reviewed the case in great detail, and wrote the paper. Dr. Goli supervised, performed literature review, provided guidance, and helped proofread the final draft.

\section{Data Availability}

The authors declare that data supporting the findings of this study are available within the article.

\section{References}

1. Dong E, Du H, Gardner L. An interactive web-based dashboard to track COVID-19 in real time. Lancet Infect Dis. 2020;20(5):533-534.

2. Cuker A, Peyvandi F. Coronavirus disease 2019 (COVID-19): Hypercoagulability. Available at: https://www. uptodate.com/contents/coronavirus-disease-2019-covid19-hypercoagulability\#!.

3. Menter T, Haslbauer JD, Nienhold R, Savic S, Hopfer H, Deigendesch N, Frank S, et al. Postmortem examination of COVID-19 patients reveals diffuse alveolar damage with severe capillary congestion and variegated findings in lungs and other organs suggesting vascular dysfunction. Histopathology. 2020;77(2):198-209.

4. Ackermann M, Verleden SE, Kuehnel M, Haverich A, Welte T, Laenger F, Vanstapel A, et al. Pulmonary vascular endothelialitis, thrombosis, and angiogenesis in
COVID-19. N Engl J Med. 2020;383(2):120-128.

5. Bilaloglu S, Aphinyanaphongs $\mathrm{Y}$, Jones $\mathrm{S}$, Iturrate $\mathrm{E}$, Hochman J, Berger JS. Thrombosis in hospitalized patients with COVID-19 in a New York City Health System. JAMA. 2020;324(8):799-801.

6. Connors JM, Levy JH. COVID-19 and its implications for thrombosis and anticoagulation. Blood. 2020;135(23):2033-2040.

7. Artifoni M, Danic G, Gautier G, Gicquel P, Boutoille D, Raffi F, Neel A, et al. Systematic assessment of venous thromboembolism in COVID-19 patients receiving thromboprophylaxis: incidence and role of Ddimer as predictive factors. J Thromb Thrombolysis. 2020;50(1):211-216.

8. Menaker J, Tabatabai A, Rector R, Dolly K, Kufera J, Lee $\mathrm{E}, \mathrm{Kon} \mathrm{Z}$, et al. Incidence of cannula-associated deep vein thrombosis after veno-venous extracorporeal membrane oxygenation. ASAIO J. 2017;63(5):588-591.

9. Gunther J. A retrospective audit on cannula-assocaited deep vein thrombosis after extracorporeal membrane oxygenation. Australian Critical Care. 2020;33(Supplement 1):S28.

10. Fisser C, Reichenbacher C, Muller T, Schneckenpointner R, Malfertheiner MV, Philipp A, Foltan M, et al. Incidence and risk factors for cannula-related venous thrombosis after venovenous extracorporeal membrane oxygenation in adult patients with acute respiratory failure. Crit Care Med. 2019;47(4):e332-e339.

11. Extracorporeal Life Support Organization (ELSO) general guidelines for all ECLS cases. Available at: https:// www.elso.org/resources/guidelines.aspx. Accessed November 5, 2020.

12. Barbaro RP, MacLaren G, Boonstra PS, Iwashyna TJ, Slutsky AS, Fan E, Bartlett RH, et al. Extracorporeal membrane oxygenation support in COVID-19: an international cohort study of the Extracorporeal Life Support Organization registry. Lancet. 2020;396(10257):10711078.

13. Beyls C, Huette P, Abou-Arab O, Berna P, Mahjoub Y. Extracorporeal membrane oxygenation for COVID19-associated severe acute respiratory distress syndrome and risk of thrombosis. Br J Anaesth. 2020;125(2):e260e262. 